\title{
\begin{tabular}{l|l} 
REVIEWERS & Genetics \\
inMedicine
\end{tabular}
}

\section{REVIEWER ACKNOWLEDGMENT 2016}

Reviewing manuscripts is arduous and time-consuming, yet it is the cornerstone of the peer-review system that advances knowledge. Genetics in Medicine would like to thank the following reviewers and editors who were kind enough to give us their time in 2016.

\begin{tabular}{|c|c|c|c|}
\hline Margaret Adam & Rebecca Chan & Steven Gray & Hane Lee \\
\hline Stacie Adams & Alka Chaubey & Robert Green & Kathleen Leppig \\
\hline Nadav Ahituv & Teresa Chen & Cheryl Greenberg & Barbara Lerner \\
\hline Joo Wook Ahn & Shilpa Chetty & Marc Greenblatt & Nancy Leslie \\
\hline Richard Allen & Wendy Chung & Scott Grosse & Ephrat Levy-Lahad \\
\hline Francesca Amati & Angus Clarke & Nelleke Gruis & Feng-Chang Lin \\
\hline Kevin Antshel & Ellen Clayton & Susanne Haga & Steve Lincoln \\
\hline Samuel Aronson & Mark Clendenning & Sihoun Hahn & Noralane Lindor \\
\hline Arthur Aylsworth & Hiram Cody & Nina Hallowell & Nicola Longo \\
\hline Daria Babushok & Janet Cragan & Alison Hamilton & Zhiyong Lu \\
\hline Richard Badge & Deborah Cragun & Emily Hardisty & Philip Lupo \\
\hline Sherri Bale & Dana Crawford & Peter Hedera & James Lupski \\
\hline Deeksha Bali & Kristy Crooks & Madhuri Hegde & Elaine Lyon \\
\hline Manisha Balwani & Robert Currier & Gail Henderson & Ebony Madden \\
\hline Michael Bamshad & Shoumita Dasgupta & Lidewij Henneman & Murugu Manickam \\
\hline Anne Bassett & Mauricio De Castro & Gail Herman & Teri Manolio \\
\hline JM Bastida & Wim de Lau & Scott Hickey & Pamela Marcus \\
\hline Linnea Baudhuin & Jantina de Vries & Jodi Hoffman & Diego Martinelli \\
\hline Christine Beck & Daniela Del Gaudio & Carol Horowitz & Monica Marvin \\
\hline Cecelia Bellcross & David Dimmock & Gabriella Horvath & Dietrich Matern \\
\hline John Belmont & Carlo Dionisi-Vici & Isa Houwink & Thomas May \\
\hline Shay ben-Shachar & Susan Domchek & Edward Hsiao & Colleen McBride \\
\hline Luisa Benussi & W. David Dotson & Louanne Hudgins & Kim McBride \\
\hline Jonathan Berg & Deb Duquette & Martina Huemer & Tomas McDonald \\
\hline Wolfgang Berger & Julie Eggington & Sara Hull & Amy McGuire \\
\hline Gerard Berry & Christine Eng & Steve Humphries & Howard McLeod \\
\hline Laura Beskow & Barbara J Evans & Katherine Hyland & Elizabeth McPherson \\
\hline Barbara Biesecker & Eric Evans & Ivan Iossifov & Susanne Meisel \\
\hline Leslie Biesecker & James Evans & Chaim Jalas & DK Meyerholz \\
\hline Patricia Birch & W. Gregory Feero & A Cecile Janssens & Jacques Michaud \\
\hline Thomas Bird & Heather Feigelson & Brian Jensen & David Miller \\
\hline Mary Helen Black & Bingjian Feng & Steve Joffe & Colleen Morris \\
\hline Kathleen Blazer & Ridgely Fisk Green & Sarah Kalia & Cynthia Morton \\
\hline Olaf Bodamer & Brent Fogel & Erik-Jan Kamsteeg & Michael Murray \\
\hline Teri Brentnall & James Ford & Peter Kang & Mitzi Murray \\
\hline Yana Bromberg & Andrea Forman & Kimberly Kaphingst & Katherine Nathanson \\
\hline Kyle Brothers & Wayne Frankel & Veronika Karcagi & Stanley Nelson \\
\hline Nicola Brunetti-Pierri & Andrea Gaedigk & Aly Karsan & Stuart Nicholls \\
\hline Adam Buchanan & Renata Gallagher & Hutton Kearney & Malgorzata Nowaczyk \\
\hline Wylie Burke & Vidu Garg & Martin Kharrazi & William Nyhan \\
\hline Michele Caggana & Masoud Garshasbi & Ian King & Julianne O’Daniel \\
\hline Ned Calonge & Gabrielle Geddes & Christopher Klein & Devin Oglesbee \\
\hline Kathleen Calzone & Bruce Gelb & Sara Knight & Joâo Paulo Oliveira \\
\hline John Carey & Santhosh Girirajan & Josh Knowles & Suzanne O’Neill \\
\hline June Carroll & Aaron Goldenberg & Ruth Kornreich & Brian O’Roak \\
\hline Gemma Carvill & David Goldgar & Dieter Kotzot & Glenn Palomaki \\
\hline Lisa Castillo & Stacy Gray & Peter Kühnen & Arti Pandya \\
\hline
\end{tabular}




\section{REVIEWERS}

$\begin{array}{llll}\text { Will Parsons } & \text { Christine Rini } & \text { Sanjay Sisodiya } & \text { Charles Venditti } \\ \text { Gregory Pastores } & \text { J. Scott Roberts } & \text { Brian Skotko } & \text { Alexandre Vieira } \\ \text { Nirali Patel } & \text { David Robertson } & \text { Leslie Smoot } & \text { Jerry Vockley } \\ \text { Melanie Pepin } & \text { Peter Robinson } & \text { Marsha Speevak } & \text { Neeta Vora } \\ \text { Susan Peterson } & \text { Kathryn Robson } & \text { Zsofia Stadler } & \text { Byron Wallace } \\ \text { Eric Pierce } & \text { Myra Roche } & \text { Robert Steiner } & \text { Bradley Walters } \\ \text { Beth Pletcher } & \text { Alan Rope } & \text { Giovanni Stevanin } & \text { Melissa Wasserstein } \\ \text { Sharon Plon } & \text { Wendy Rubinstein } & \text { Dave Stevenson } & \text { Michael Watson } \\ \text { Joachim Pohlenz } & \text { Katie Rudd } & \text { Elena Stoffel } & \text { Yvonne Weber } \\ \text { Leona Poon } & \text { Marcia Russell } & \text { Charles Strom } & \text { Karen Weck } \\ \text { Lori Potocki } & \text { Sandra Ryeom } & \text { Amy Sturm } & \text { David Weinstein } \\ \text { Beth Potter } & \text { Avni Santani } & \text { V. Nathan Subramaniam } & \text { James Weisfeld-Adams } \\ \text { Bradford Powell } & \text { Morten Scheibye-Knudsen } & \text { Vernon Sutton } & \text { Rosanna Weksberg } \\ \text { Cynthia Powell } & \text { Maren Scheuner } & \text { Eric Swindell } & \text { Georgia Wiesner } \\ \text { Barbara Prainsack } & \text { Fredrick Schumacher } & \text { Laura Tafe } & \text { Robert Wildin } \\ \text { Aleix Prat } & \text { Marc Schwartz } & \text { Jonathan Tait } & \text { Janet Williams } \\ \text { Thomas Prior } & \text { Siby Sebastian } & \text { Ran Tao } & \text { Marc Williams } \\ \text { Reed Pyeritz } & \text { Alvaro Serrano Russi } & \text { Nicholas Taylor } & \text { Michael Wilschanski } \\ \text { Ros Quinlivan } & \text { Brian Shaffer } & \text { Steven Teutsch } & \text { Lee-Jun Wong } \\ \text { Nadeem Qureshi } & \text { Suma Shankar } & \text { Erik Thorland } & \text { Alan Wright } \\ \text { Alanna Rahm } & \text { Richard Sharp } & \text { Brad Tinkle } & \text { Chao Xing } \\ \text { Katherine Rauen } & \text { Chad Shaw } & \text { Karen Tsuchiya } & \text { Xianyong Yin } \\ \text { Victoria Raymond } & \text { Eliot Shearer } & \text { Nadine Tung } & \text { Joon-Ho Yu } \\ \text { Kavita Reddy } & \text { Paul Shekelle } & \text { Sandra van Calcar } & \text { Ulrich Zanger } \\ \text { Katie Reeder-Hayes } & \text { Brian Shirts } & \text { Ignatia Van den Veyver } & \text { Maimoona Zariwala } \\ \text { Heidi Rehm } & \text { D. Sidjanin } & \text { Anthony Vandersteen } & \text { Barbara Zehnbauer } \\ \text { Bronson Riley } & \text { Rani Singh } & \text { Jason Vassy } & \end{array}$

If you reviewed for us in 2016 and are not on this list, please contact the managing editor, Jan Higgins, at gim@acmg.net and we will include you in our next issue. 